\title{
Guías clínicas para la cirugía de la epilepsia y de los trastornos del movimiento
}

\author{
Grupo de Cirugía Funcional de la Sociedad Española de Neurocirugía (SENEC)
}

\section{Resumen}

Las guías clínicas para la cirugía de los trastornos del movimiento y de la epilepsia han sido diseñadas y consensuadas por el grupo de cirugía funcional de la Sociedad Española de Neurocirugía (SENEC). Las guías son recomendaciones para la realización de ambas cirugías y el formato sobre el que están basadas son los estudios prospectivos basados en evidencia científica así como en la opinión de los componentes del grupo.

PALABRAS CLAVE: Epilepsia. Trastornos del movimiento. Cirugía.

Guidelines for the surgical treatment of the movement disorders and epilepsy

\section{Summary}

The guidelines for the surgical treatment of the movement disorders and epilepsy have been performed by the functional and stereotactic group of the Spanish Society of Neurosugery (SENEC). The guidelines are recomendations in terms of indication for surgery including timing and methods. The format are supported by prospective studies based in scientific evidence and the expert opinion of the group.

\section{KEY WORDS. Epilepsy. Movement disorders. Surgery.}

Las guías clínicas para la cirugía de los trastornos del movimiento y de la epilepsia han sido elaboradas y consensuadas por el grupo de cirugía funcional de la Sociedad. Están basadas en evidencia científica así como en la experiencia clínica. La utilidad de las mismas se dirige a neurocirujanos, grupos de trabajo y centros que están realizando o bien quieren empezar este tipo de cirugía. El objetivo de las mismas es el de proponer y exigir un nivel básico pero imprescindible para la realización de cual-

Recibido: Recibido: 6-10-08. Aceptado: 2-12-08 quiera de las cirugías referidas en el campo de la epilepsia y de los trastornos del movimiento.

Las mismas no se contemplan como de obligatorio cumplimiento sino que son de una clara "recomendación" para los distintos grupos quirúrgicos. El grupo de cirugía funcional de la SENEC cree que pueden ser útiles para otras sociedades quirúrgicas, científicas e incluso para entidades oficiales tanto a nivel de comunidades autónomas como a nivel nacional. Los procedimientos quirúrgicos tanto para la cirugía de la epilepsia como de los trastornos del movimiento deben de estar dirigidos por neurocirujanos y no deben de ser utilizados por personas, sociedades o entidades que no pertenezcan al ámbito neuroquirúrgico. También el grupo cree que en un momento determinado las guías clínicas para ambos tratamientos pudieran tener un valor legal. En las mismas se exige la creación de grupos de trabajo con expertos en distintos campos para la mejor realización y resultados de estos tipos de cirugías.

Guía Clínica de la cirugía para tratamiento de los trastornos del movimiento

\section{Necesidades mínimas del grupo para la realización de cirugía funcional:}

Formación de un grupo de trabajo con un neurocirujano con conocimiento de ganglios basales y de cirugía estereotáctica funcional.

Necesidad de un especialista en registro cerebral profundo en el grupo (neurofisiólogo/neurólogo).

Selección del paciente por neurólogos especialistas en el campo de los trastornos del movimiento.

\section{Selección del candidato quirúrgico} Enfermedad de Parkinson (EP).

Pacientes con complicaciones motoras (fluctuaciones y disquinesias) y buena respuesta a la medicación.

Pacientes intolerantes a dopa.

Edad máxima quirúrgica a determinar por cada grupo.

No demencia ni problemas cognitivos severos.

No otras complicaciones que impidan cirugía estereo- 
táctica (problemas cardiológicos, EPOC, HA descompensada, atrofia cortical o ventriculomegalia, otros hallazgos en RMI preoperatoria, etc)

Distonía

Distonía primaria y RMI sin lesiones

\section{Temblor}

Temblor postraumático, esencial, esclerosis múltiple o temblor de Holmes. Incapacidad para hacer vida normal. No respuesta a tratamientos médicos.

\section{Selección de la diana quirúrgica}

Vim: Temblor esencial, postraumático y por esclerosis múltiple. Temblor parkinsoniano?

$C m / P f:$ Gilles de la Tourette

$G P i$ : EP. Distonía. Hemicorea/Hemibalismo. Gilles de la Tourette.

NST: EP

Otras dianas como zona incerta o el núcleo pedúnculopontino (PPN) para la EP o el NST para la distonía no están lo suficientemente probadas como dianas eficaces en estudios clínicos y actualmente no pueden ser recomendados fuera de los estudios de investigación clínica.

\section{Requisitos quirúrgicos de un grupo de cirugía de gan- glios basales}

Protocolización de la Imagen (RMI, TAC, Fusión, etc)

Protocolización de la diana quirúrgica

Protocolización del registro fisiológico.

Protocolización de la implantación del electrodo.

Vigilancia intensiva postoperatoria.

Protocolización de prueba de imagen postoperatoria.

Implantación de batería en un primer o segundo tiempo con anestesia general.

Protocolización de estimulación por parte de neurólogo con experiencia

Deseable la realización de 12 cirugías anuales por mantenimiento del equipo y disminución de complicaciones quirúrgicas.

\section{Otros tratamientos de la EP}

Recomendaciones sobre implantes dopaminérgicos como no dopaminérgicos (GDNF, cuerpo carotídeo, células madre, otros tejidos) están actualmente en fase experimental y no lo suficientemente demostrados para ser aplicados en clínica. Cualquier tratamiento debe realizarse dentro de estudios de investigación y no actualmente estudios clínicos.

\section{Guía clínica en cirugía de la epilepsia}

\section{Introducción}

La cirugía es una opción terapéutica infrautilizada en el tratamiento de las distintas formas de epilepsia refractaria al tratamiento farmacológico. Las crisis fármaco resistentes con deterioro de la calidad de vida pueden ser curables o controlables mediante cirugía.

La epilepsia es una de las enfermedades neurológicas crónicas más comunes, con una prevalencia aproximada del $0.5-1 \%$ (unas 350.000 personas en España) y una incidencia acumulada a lo largo de la vida en torno al 3\%. Aproximadamente un $60 \%$ de todas las epilepsias tienen un origen focal, es decir, se originan dentro de una región cerebral circunscrita de la corteza cerebral. De entre las epilepsias focales, el tipo más frecuente está constituido por aquéllas que tienen su origen en el lóbulo temporal (55\%). En la actualidad, la epilepsia focal es el único tipo de epilepsia curable.

El principal objetivo en el tratamiento de los pacientes con epilepsia es conseguir un adecuado control de sus crisis, mejorando así su calidad de vida y evitando el deterioro y la exclusión asociados a la presencia de crisis epilépticas frecuentes y mantenidas a lo largo de años. La primera aproximación terapéutica será la utilización de fármacos antiepilépticos. A pesar de la introducción de nuevos fármacos para el control de las crisis con múltiples mecanismos de acción diferentes, el tratamiento farmacológico consigue controlar adecuadamente las mismas en menos de un $80 \%$ de los pacientes. Un $20 \%$ de los pacientes con epilepsia no responde al tratamiento farmacológico. El 75\% del gasto generado por la epilepsia en España se concentra en este grupo de pacientes. Los individuos que resulten ser resistentes al tratamiento farmacológico deben ser remitidos a una unidad de epilepsia para la realización de una valoración prequirúrgica. Aproximadamente la cuarta parte de los pacientes fármaco resistentes podrían beneficiarse del tratamiento quirúrgico, lo que para España supone una estimación de unos 20.000 candidatos a cirugía de la epilepsia. En la actualidad, sin embargo, sólo un pequeño porcentaje de los candidatos quirúrgicos potenciales es remitido a las unidades de cirugía de la epilepsia.

La cirugía de la epilepsia actual requiere de un equipo multidisciplinario de especialistas con una formación específica en la materia, de su colaboración en una misma unidad, y de los medios tecnológicos necesarios. No hay un acuerdo unánime acerca de qué tipo y cantidad de información se debe obtener antes de indicar un tipo concreto de intervención quirúrgica, de entre las distintas opciones técnicas empleadas. No obstante, los protocolos de los distintos centros presentan un mayor consenso a medida que se acumulan e interpretan las evidencias sobre los resultados obtenidos en la cirugía de la epilepsia.

\section{Resistencia a fármacos, cronicidad y gravedad}

Serán considerados como fármaco resistentes aquellos pacientes en los que no se haya conseguido el control de sus crisis tras dos años de utilización de dos fármacos de primera línea en monoterapia y, al menos, una asociación en 
biterapia a las máximas dosis toleradas. Las crisis deben ser causa de un deterioro de la calidad de vida del paciente.

\section{Selección del candidato a cirugía}

Una vez se ha determinado el diagnóstico de epilepsia resistente a tratamiento farmacológico se considerarán candidatos a evaluación prequirúrgica aquellos pacientes que cumplan los siguientes criterios:

1. Ausencia de enfermedades neurológicas progresivas (tumores cerebrales malignos, esclerosis múltiple, vasculitis, etc.).

2. La duración de la epilepsia debe de ser de al menos uno ó dos años. Sin embargo, se permiten excepciones en función de la patología cerebral subyacente (epilepsia sintomática, esclerosis temporal medial), de la intensidad semiológica de las crisis, de su frecuencia y de la gravedad del deterioro personal y del aislamiento social, educativo o laboral al que sometan al paciente. También casos pediátricos pueden considerarse periodos más cortos para detener la alteración del desarrollo neuropsicológico causado por los fármacos antiepilépticos y las crisis repetidas.

3. Limitación manifiesta de la calidad de vida debido a la presencia de las crisis epilépticas.

4. El paciente debe entender y aceptar la posibilidad de requerir tratamiento farmacológico tras la cirugía.

5. La presencia de enfermedades psiquiátricas ó médicas serán una contraindicación para la cirugía cuando éstas supongan un riesgo evidente para el paciente, bien durante la evaluación prequirúrgica o bien durante la cirugía.

\section{Evaluación prequirúrgica}

La intervención quirúrgica óptima para el tratamiento de la epilepsia es la que destruye la cantidad justa de tejido neural para eliminar las crisis sin provocar déficits neurológicos añadidos.

- Por lo tanto, los objetivos de la evaluación prequirúrgica son:

1. Identificar la localización y extensión de la zona epileptógena. El concepto teórico de zona epileptógena se define como el área de tejido cerebral necesario y suficiente para originar las crisis epilépticas, y cuya extirpación o desconexión conduce a la supresión de las mismas.

2. Valorar el impacto potencial de la cirugía sobre la función neurológica, el rendimiento cognitivo, y el estado emocional del paciente.

- Métodos de evaluación prequirúrgica: no disponemos de una prueba sencilla que permita por sí misma delimitar la llamada zona epileptógena. Los límites de la zona epileptógena sólo pueden definirse por aproximación, identificando aquellas áreas cerebrales caracterizadas por una disfunción persistente, tanto epiléptica como no epiléptica. Con ese propósito se emplea la amplia variedad de pruebas diagnósticas que se enumeran a continuación.

\section{Identificación de la zona epileptógena}

La localización y extensión de la zona epileptógena se deduce de la determinación de una serie de zonas corticales de interés durante la evaluación prequirúrgica:
1. Zona sintomatogénica
2. Zona irritativa / Zona de inicio ictal
3. Zona lesional epileptogénica
4. Zona funcional deficitaria

\section{Zona sintomatogénica}

Se define como la región cortical que cuando es activada por una descarga epiléptica reproduce los síntomas/signos clínicos del paciente. La zona sintomatogénica que produce los síntomas iniciales es normalmente la más importante como indicador de la localización del área epileptogénica.

La zona sintomatogénica se determina mediante:

1. Historia clínica

2. Monitorización video-EEG: exploración fundamental obligada y eje de toda evaluación prequirúrgica en cirugía de la epilepsia.

$\square$ Permite el estudio detallado y sistemático de los síntomas y signos críticos.

- Ayuda a confirmar el diagnóstico de epilepsia en aquellos casos en los que no se puede llegar a una conclusión definitiva a través de la historia clínica.

$\square$ Clasifica los distintos tipos de crisis y verifica la frecuencia y los factores desencadenantes de las mismas.

$\square$ Determina de forma precisa la actividad epileptiforme intercrítica (zona irritativa) y del patrón crítico (zona de inicio ictal).

\section{Zona irritativa / zona de inicio ictal}

2.1. Zona Irritativa: región de la corteza cerebral que genera la actividad epiléptica intercrítica en el EEG de superficie. La localización de la actividad intercrítica es un buen indicador de la zona epileptógena. Habitualmente la zona irritativa y la zona epileptógena no se solapan, siendo la zona irritativa más extensa que la zona epileptogénica.

La zona irritativa se delimita mediante:

a) Monitorización video-EEG

b) Monitorización INVASIVA, o registro de la actividad epiléptica mediante electrodos intracraneales de manera prolongada: electrodos de foramen oval, electrodos subdurales, electrodos intracerebrales profundos.

c) Magneto encefalografía, si se dispone de ella.

2.2. Zona de inicio ictal: región de la corteza en la que las crisis tienen su origen. La zona de inicio ictal y la zona epileptogénica no necesariamente se superponen. No obstante, la zona de inicio ictal es el mejor marcador de la localización de la región epileptógena.

Métodos para determinar la zona de inicio ictal: 
a) Monitorización video-EEG: registro simultáneo de la actividad eléctrica cerebral y de la semiología clínica. Por lo general en régimen de ingreso hospitalario y durante un periodo de tiempo determinado por el número de crisis epiléptica típicas registradas.

b) Monitorización INVASIVA, o registro de la actividad epiléptica mediante electrodos intracraneales de manera prolongada: Electrodos de foramen oval, electrodos subdurales, electrodos intracerebrales profundos.

c) Neuroimagen Funcional:

$\square$ La tomografía computarizada por Emisión de Fotón Único (SPECT) cerebral de perfusión con HMPAO durante la crisis (SPECT ictal).

$\square$ Superposición sobre la Resonancia Magnética (RM) de la imagen resultante de la sustracción entre el SPECT ictal y el intercrítico (SISCOM).

$\square$ RM funcional con técnica BOLD (Blood Oxygenation Level Dependant), que determina los patrones de activación cortical de manera indirecta.

\section{Zona lesional epileptógena}

Alteración estructural visible en la neuroimagen estructural, especialmente en la RM craneal. Todo paciente con crisis epilépticas debe ser sometido a una RM craneal. En algunos casos, puede ser necesario complementarla con tomografía computarizada o angiografía digital. La ausencia de lesión en un examen convencional de RM craneal no descarta la misma. Se recomienda la práctica de una resonancia magnética (RM) de alta resolución (1.5T o 3T) siguiendo un protocolo especial para pacientes con epilepsia.

Es fundamental establecer la relación entre la lesión estructural y la generación de las crisis epilépticas. En especial en caso de lesiones múltiples, de lesiones extensas o de hallazgos aparentemente incidentales.

\section{Zona deficitaria funcional}

Área cortical de funcionamiento anómalo durante el periodo intercrítico, bien como efecto de una la lesión, bien como resultado de una transmisión sináptica alterada. La zona deficitaria orienta acerca de la localización zona epileptógena. Se delimita mediante:

- Exploración neurológica

- Evaluación neuropsicológica

- EEG intercrítico

- SPECT intercrítico: área de hipoperfusión

- Tomografía por emisión de positrones (PET) interictal con 2- fluoro-2-desoxiglucosa: hipometabolismo.

\section{Valoración de las secuelas potenciales de la cirugía}

La evaluación de las secuelas funcionales previsibles se basa en la localización anatómica y la correlación funcional de la zona a resecar, corroborada mediante las técnicas de cartografía funcional de la corteza cerebral

La RM funcional permite en la actualidad la evaluación de las áreas funcionales primarias. En los casos en que la información aportada por la RM funcional sea insuficiente o poco fiable, será necesario recurrir al "mapping" cortical intraoperatorio o con electrodos subdurales implantados.

De manera análoga, la evaluación de las secuelas cognitivas potenciales requiere de exploración neuropsicológica detallada. Si los resultados de las técnicas no invasivas de evaluación neuropsicológica (exámenes neuropsicológicos, RM funcional de lenguaje) planteen dudas o sean insuficientes, se emplearán técnicas invasivas de evaluación neuropsicológica, incluyendo el test del amobarbital intracarotídeo, que permiten modificar la estrategia quirúrgica cuando predicen que una intervención puede llegar a causar déficits añadidos de memoria verbal u otras funciones neurológicas.

La evaluación psiquiátrica forma parte de la evaluación prequirúrgica de cualquier paciente admitido en la unidad de epilepsia para estudio prequirúrgico. Existe una elevada prevalencia de patología psiquiátrica comórbida en pacientes con epilepsia refractaria a tratamiento farmacológico. Los trastornos más frecuentemente asociados son: trastornos del ánimo y del comportamiento. Estos trastornos tienen un impacto determinante en la calidad de vida de los pacientes con epilepsia y, por tanto, deben ser adecuadamente evaluados y tratados.

La evaluación psiquiátrica será necesaria, también, una vez realizada la cirugía para poder valorar las posibles complicaciones psiquiátricas que puedan surgir tras la misma. Los trastornos más frecuentes son: del estado de ánimo (depresión-50\% de los pacientes en los primeros seis meses), labilidad emocional y psicosis. Estos trastornos aparecen con más frecuencia en aquellos pacientes que los presentaban o presentaron en algún momento previo a la cirugía. Por tanto, la evaluación prequirúrgica podría servirnos como indicador de qué sujeto está más expuesto a presentar complicaciones psiquiátricas tras la cirugía.

\section{Tratamiento quirúrgico. Procedimientos reglados}

Existen tres síndromes epilépticos cuyo tratamiento de elección es neuroquirúrgico: la epilepsia temporal medial, las lesiones neocorticales delimitadas y las enfermedades hemisféricas difusas. Son las tres entidades clínicas de las que se conoce en buena parte tanto su fisiopatología como historia natural. Su pronóstico es francamente malo cuando se aplica únicamente tratamiento médico, mientras que responden bien al tratamiento quirúrgico. Los pacientes afectos por dichos síndromes pueden ser identificados con relativa facilidad y se trata de enfermedades de carácter progresivo, por lo que deben ser remitidos a una unidad de cirugía de la epilepsia tan pronto como la medicación 
antiepiléptica de primera línea se muestre ineficaz.

Epilepsia temporal medial: Es el prototipo de síndrome susceptible de tratamiento neuroquirúrgico, y el único en el que la eficacia terapéutica superior de la cirugía respecto del tratamiento farmacológico ha sido demostrada por un estudio con un grado de evidencia Clase I (Wiebe. NEJM, 2001). Tiene una presentación clínica característica y una base fisiopatológica específica: la esclerosis del hipocampo. En algunos casos, sin embargo, puede estar en relación con lesiones neoplásicas o malformativas de dicha región. Posiblemente es la forma más común de epilepsia al tiempo que una de las más refractarias al tratamiento médico.

En la actualidad se practican diversas variantes técnicas de resección del lóbulo temporal. En la mayoría de casos la resección se basa en indicadores preoperatorios de patología del hipocampo no invasivos, más que en los hallazgos intraoperatorios intercríticos de la electro-corticografía. El objetivo común de todas ellas es la extirpación del sustrato patológico de la epilepsia temporal medial, mientras que las diferencias residen tanto en el grado de exéresis considerado necesario y suficiente para la curación, como en las estrategias para evitar complicaciones quirúrgicas y déficits postoperatorios. No obstante, los resultados en cuanto a eficacia y seguridad no difieren de manera significativa.

La resección temporal anteromedial es probablemente la variante técnica más extendida en el momento presente, tanto por su seguridad como por sus buenos resultados en el control de las crisis. Se trata de una resección en dos tiempos. El primer tiempo consiste en la resección en bloque de $3,5 \mathrm{~cm}$. del polo temporal por debajo de la primera circunvolución temporal. En el segundo tiempo se lleva a cabo una exéresis subpial de las estructuras mediales: hipocampo, corteza entorrinal-parahipocampo y núcleo amigdalino, al tiempo que queda intacto el neocórtex lateral y la mayor parte de las radiaciones ópticas. El límite medial de la resección es la cisterna ambiens, y el límite posterior se extiende hasta la curvatura de la cola del hipocampo, a la altura del tubérculo cuadrigémino superior. Ello supone una resección hipocámpica de unos $3 \mathrm{~cm}$ de longitud.

Lesionectomía-corticectomía: indicadas en crisis parciales refractarias causadas por lesiones estructurales en el neocórtex, como malformaciones congénitas (especialmente trastornos del desarrollo cortical) o tumores neurogliales. La evaluación y decisión quirúrgica en estos casos requiere una cautela especial, ya que algunas lesiones estructurales no son valorables desde un punto de vista clínico y otras pueden formar parte de una patología multifocal, en la cual otra lesión no detectada puede ser la verdadera causa de la epilepsia. En consecuencia, el tratamiento quirúrgico no debe llevarse a cabo sobre la base única de la imagen estructural, sino que es necesario confirmar la epileptogenicidad de la lesión, lo que equivale en la mayoría de las ocasiones al estudio del EEG crítico, o al estudio electrocorticográfico crítico si los resultados del estudio preoperatorio así lo indican.

Hemisferotomía (hemisferectomía funcional): Algunos de los síndromes epilépticos catastróficos focales o generalizados que aparecen en niños y lactantes pueden tener su origen en enfermedades confinadas en un hemisferio cerebral o parte de éste. En este grupo se incluyen la hemimegalencefalia y otras displasias corticales difusas, el síndrome de Sturge-Weber, los quistes porencefálicos de gran tamaño y la encefalitis de Rasmussen. La evaluación preoperatoria de estos pacientes permite en muchos casos demostrar que las anomalías epileptógenas quedan confinadas a un solo hemisferio, mientras que el contralateral mantiene su función relativamente intacta. En estas situaciones, la hemisferotomía puede controlar las crisis habituales y detener el inevitable retraso psicomotor.

La hemisferectomía anatómica convencional ha sido abandonada en la actualidad, a causa de las graves complicaciones tardías asociadas, en concreto la hemosiderosis superficial, y ha sido sustituida por la hemisferotomía. La hemisferotomía se realiza por vía intraventricular a través de un abordaje periinsular y permite la desconexión funcional completa del hemisferio, al tiempo que preserva su aporte vascular e integridad anatómica.

Debido a que la hemisferotomía se practica por lo general sólo en aquellos pacientes que ya han desarrollado una hemiparesia grave con pérdida de función en la mano, estas intervenciones no causan déficit motor añadido. De hecho, la función de las extremidades afectas a menudo mejora si se practica a edades tempranas. Sin cirugía estos niños suelen verse condenados a profundas minusvalías y a una institucionalización de por vida, mientras que si la intervención se lleva a cabo en el momento oportuno la probabilidad de desarrollar una vida casi normal puede alcanzar el $60-80 \%$.

\section{Procedimientos paliativos}

Callosotomía: los pacientes que sufren epilepsia secundariamente generalizada, como los afectos por el síndrome de Gastaut-Lennox, presentan daño cerebral difuso y una elevada frecuencia de crisis atónicas con caídas -drop attacks- que provocan traumatismos craneo-encefálicos repetidos. La medicación antiepiléptica suele ser ineficaz contra estas crisis. La callosotomía puede considerarse como la opción quirúrgica para los enfermos en los que los drop attacks constituyan el tipo de crisis más invalidante. La técnica actual de la callosotomía limita la sección del cuerpo calloso a los dos tercios anteriores de dicha comisura mayor, ya que se ha demostrado suficientemente efectiva en la mayoría de casos y está gravada con una menor incidencia de síndromes posquirúrgicos de desconexión y deterioro neuropsicológico. Si fracasa en el control de las crisis la callosotomía puede completarse en una segunda 
intervención.

La sección del cuerpo calloso no es una intervención curativa, aunque puede obtener la desaparición de los drop attacks en una proporción de casos apreciable, logrando una mejoría significativa en el $70 \%$ de los intervenidos. Dado su carácter paliativo, la callosotomía no suele mejorar los otros tipos de crisis, ni el déficit neurológico ni el deterioro cognitivo habitualmente asociado a la epilepsia secundariamente generalizada. Por todo ello, la tendencia actual es -previamente a la callosotomía- ensayar la estimulación vagal, más segura y de eficacia clínica similar (ver más adelante).

Transición subpial múltiple (TSM): tiene por objetivo la interrupción quirúrgica de las fibras horizontales intracorticales dentro de la zona epileptógena. La técnica consiste en practicar transecciones subpiales verticales y paralelas, separadas $5 \mathrm{~mm}$ entre sí. Se basa, por una parte, en que interfiere el papel fundamental que juegan las fibras horizontales intracorticales en la sincronización neuronal, en la aparición de descargas paroxísticas y en su propagación; mientras que por otra parte respeta la estructura columnar vertical del córtex, previniendo así la aparición de déficits postquirúrgicos. La TSM se propone como una técnica alternativa a la resección de la zona epileptógena cuando ésta se halla en un área de elevada elocuencia y en la cual, la práctica de una resección cortical conllevaría secuelas neurológicas inaceptables. Este sería el caso, por ejemplo, de la agnosia verbal progresiva que afecta a los niños con síndrome de Landau-Kleffner, supuestamente causada por actividad epiléptica criptogenética que afecta a la corteza del lenguaje. Los resultados más recientes de las mejores series, con un seguimiento mínimo de 4 años, muestran un $55 \%$ de curaciones con un $4 \%$ de déficits permanentes.

Estimulación vagal (VNS): la estimulación eléctrica del nervio vago se basa en datos experimentales que indican su capacidad para reducir la frecuencia y gravedad de ciertos tipos de crisis parciales. Su indicación habitual es la epilepsia parcial grave no susceptible de cirugía resectiva y como alternativa o complementaria a la callosotomía. Técnicamente consiste en la implantación de un electrodo espiroideo en el trayecto cervical del nervio vago izquierdo, conectado a un generador subcutáneo que emite impulsos eléctricos cuyos parámetros más eficaces aún están por establecer de manera definitiva. La mitad de los pacientes sometidos a VNS muestra una respuesta terapéutica en forma de una reducción de la frecuencia media de las crisis del $50 \%$.

Estimulación cerebral profunda (ECP): dentro de las aplicaciones de la estimulación neural en el tratamiento de las formas más graves de epilepsia fármaco resis- tente, cabe citar la estimulación eléctrica de estructuras subcorticales, como el núcleo centromediano del tálamo, el núcleo subtalámico de Luys o el núcleo anterior del tálamo. Se trata de un procedimiento paliativo cuyo propósito es reducir la epileptogenicidad cerebral. Las indicaciones todavía no se han delimitado y los resultados clínicos son aún preliminares, pero indican que puede ser efectiva en la reducción del número de ataques en pacientes con epilepsia fármaco resistente no susceptible de cirugía resectiva.

Seguimiento. El seguimiento mínimo tras la cirugía debería ser de dos años, aunque se recomienda un seguimiento medio de cinco años. Los controles son periódicos, con frecuencia decreciente si no hay eventualidades. Por lo general el seguimiento es llevado a cabo por los distintos especialistas integrantes del equipo de epilepsia:

- Evolución post-quirúrgica propiamente dicha, incluyendo neuroimagen

- Resultado en cuanto a las crisis mediante escalas validadas (ILAE, Engel) y pauta de medicación antiepiléptica evaluación del estado neuropsicológico y rehabilitación si procede

- Control del estado emocional y de posibles trastornos psiquiátricos

- Resultado en cuanto a calidad de vida (escalas QOLIE).

A la hora de valorar la relación coste/beneficio de la cirugía de la epilepsia es esencial traducir el éxito clínico que supone la supresión de las crisis en términos de rehabilitación psicosocial, eliminación de incapacidades y mejoría de la calidad de vida. Aunque la curación de las crisis en un paciente suponga un ahorro importante en cuanto a gasto sanitario directo, una parte de los intervenidos puede continuar dependiendo de sus familias o de la administración pública, generando un elevado coste indirecto asociado a su invalidez.

El grupo de Cirugía Funcional de la SENEC que ha participado en estas guías está formado por los Drs: J.A. Aparicio; J.A. Barcia; R. García de Sola; F. García Salazar; J. Molet; F. Robaina; R. Rumiá; F. Seijo; E. García Navarrete; J. Albisua; M. Gelabert; E. Ramos; G. Bilbao; M. Manrique; J.A. Alberdi; M. Katati; R. Martínez; C. Botella; J. Muñoz; J.M. Montero; J.F. Paz; P. Roldán; G. García March; M. del Álamo; J. Ayerbe; J. Guridi: Guías clínicas para la cirugía de la epilepsia y de los trastornos del movimiento. Neurocirugía 2009; 20: 329-334.

Correspondencia postal. Dr Jorge Guridi Coordinador del grupo de cirugía Funcional de la SENEC. Servicio de Neurocirugía Clínica Universitaria Universidad de Navarra. 31008 Pamplona. 